\title{
Оценка эффективности сделок слияний и поглощений: интегрированная методика
}

\author{
Хусаинов 3.И. ${ }^{4}$
}

В работе исследуется изменение котировок и финансовых результатов компаний при сделках слияний и поглощений. Анализ значительного массива сделок с публичными компаниями с 2005 года во всем мире показывает взаимосвязь между реакцией рынка на информацию о сделке и последующей динамикой финансовых показателей объединенной компании через два года после сделки. Степень связи варьируется в зависимости от развитости фондовых рынков, размеров компании и прочих качественных показателей.

JEL: G14; G15; G24; G34

Ключевые слова: сделки, слияния и поглощения, акциии, котировки, рыночная эффективность

\section{Введение}

В последнее время в мире, в том числе и в России, наблюдается стремительное увеличение числа и объемов сделок слияний и поглощений. Интеграционные процессы изменяются структурно, в них вовлекается все большее число регионов и расширяются масштабы межнациональных сделок. Для бизнес-сообщества данные процессы имеют ясную логику, поскольку предполагают очевидную экономическую мотивацию: расширение рынков сбыта, производственную синергию, финансовые выгоды, то есть факторы, ведущие к увеличению стоимости собственного капитала.

Однако для исследователей данная тема до сих пор остается незавершенной. Вопервых, несмотря на большое количество работ, наблюдается очевидный пробел в качественных выводах, и эта проблематика в классических работах по корпоративным финансам относится к нерешенным проблемам финансов [Brealey, Myers, 1991]. Bo-вторых, в научном сообществе устоялось мнение о том, что интеграционные сделки в большинстве случаев ведут к разрушению акционерного капитала: котировки акций компании-покупателя падают; финансовые результаты объединенной компании ухудшаются, что противоречит наблюдающейся интеграционной активности.

Данная ситуация тем более удивительна, что существует множество эмпирических работ, авторы которых приходят к противоположным выводам ввиду различий в предпосылках, методах анализа, исследуемой выборки сделок. Бесспорно, существующее несоответствие научных выводов и практических реалий требует разрешения. Очевидно, что исследуемая проблема крайне актуальна, поскольку эффективности нарастающего интеграционного процесса необходима качественная, фундаментальная научная база.

Все существующие исследования по подходу к измерению результативности слияний и поглощений можно условно разделить на четыре группы [Beitel, Schiereck, Wahrenburg, 2002].

1. Изучение доходности акций.

Данные работы, составляющие большинство исследований в данной отрасли, рассматривают аномальную доходность акций компаний в период появления информации о сделке. Преимущество метода заключается в непосредственном анализе влияния сделки на благосостояние акционеров.

2. Анализ финансовой отчетности.

\footnotetext{
${ }^{4}$ Аспирант кафедры экономики и финансов фирмы ГУ ВШЭ.

Выпуск \#1(5), 2008 () Электронный журнал Корпоративные Финансы, 2008
} 
Подход основывается на финансовой отчетности до сделки и после нее, фокусируясь на различных относительных показателях. Как правило, в качестве меры сравнения используются аналогичные неслившиеся компании.

3. Опросы менеджеров.

Подход анализирует результаты опросов менеджеров, касающихся результатов сделки. Как правило, выводы, полученные из стандартизованных вопросников, обобщаются на всю выборку.

4. Case studies.

Данные работы фокусируются на одной сделке или их ограниченной выборке, используя в качестве базы для анализа данные глубоких интервью менеджеров и аналитиков. Данный метод очень продуктивен тем, что детальное изучение вопроса может открыть новые, ранее не изучаемые аспекты проблемы.

Изучение доходности акций. Данный подход основан на рассмотрении доходности акций до и после объявления о сделке. Доходность для одного дня рассчитывается как отношение изменения курса акции и выплаченных дивидендов к цене акций за день до опубликования информации. Аномальной считается любая доходность, которая превышает обычную для этой ценной бумаги. Под обычной доходностью, как правило, понимается доходность согласно модели САРМ или доходность крупного рыночного индекса, например S\&P500.

Данные исследования основаны на предположении о том, что в ценах акций отражена приведенная стоимость всех ожидаемых будущих денежных потоков. Данный подход начиная с 70-х годов доминировал в исследованиях данной тематики.

Преимуществом данного способа является то, что он позволяет напрямую измерять добавочную стоимость, создаваемую для инвесторов. Также предполагается, что данный подход устремлен в будущее, оценивая предполагаемые денежные потоки.

С другой стороны, это требует очень строгих предположений о работе фондовых рынков. В частности, эффективности, рациональности инвесторов и отсутствии ограничений для арбитража. Есть предположения, что данные требования выполняются не всегда и не для всех акций.

К тому же при измерении изменения в курсе цен наблюдаются очень большие помехи от других событий компании, что может сильно исказить наблюдаемую доходность. Благо закон больших чисел во многом позволяет нивелировать данный эффект.

Данные исследования можно условно разделить на анализ доходности для владельцев акций поглощаемой компании и покупающей компании.

Доходность для акционеров поглощаемой компании. Большинство исследований показывает, что владельцы покупаемой компании, как правило, получают значительную положительную доходность. Анализ работ показывает, что положительная доходность не зависит от условий сделки, временного горизонта и прочих факторов. В среднем аномальная доходность достигает 20-30\%. Можно утверждать, что акционеры поглощаемой компании, как правило, получают премию.

Доходность для акционеров компании-поглотителя. Данная сфера исследования является самой проблематичной. В каждом новой работе дается новая оценка данного явления. Можно утверждать, что нет ни одного исследования, которое представляло бы бесспорные результаты, безоговорочно признаваемые всеми.

Часть исследователей утверждают наличие отрицательной доходности для компаниипокупателя, сопоставимое количество других утверждают обратное. Такое значительное разнообразие результатов легко объясняется как существенным различием анализируемой выборки, так и применяемой методологией.

Различные авторы в своих работах пытаются выделить определенные закономерности для различных сегментов генеральной совокупности сделок. 
Во-первых, существует комплекс работ, изучающих лишь крупные сделки. Классическая работа Хейли, Палепу и Рубак [Healy, Palepu\&Ruback, 1992] анализирует лишь крупнейшие американские сделки 80-х годов.

Во-вторых, можно выделить работы, исследующие сделки по географическому принципу. Так, например, Экбо и Тормбурн [Eckbo\&Thorburn, 2000] изучают канадские, Реннебуг и Гоерген [Renneboog\&Goergen, 2003] — европейские, а Куиперс, Миллер и Патель [Kuipers, Miller\&Patel, 2002]- интернациональные сделки.

В-третьих, широко распространено изучение слияний в определенных отраслях. Бейтель [Beitel, 2002] рассматривает банки, а Макиейра, Меггинсон и Нейл [Maquieira, Megginson\&Nail, 1998], в свою очередь, конгломератные сделки.

В-четвертых, исследователи изолируют выборку определенными типами сделок. Йаррель и Полсен [Jarrel\&Poulsen, 1989] анализируют только поглощения, Баннерйе, Оуверс [Bannerjee, Owers, 1992] - сделки с «белыми рыцарями», Мильерин [Mulherin, 2000] - только незавершенные сделки.

Очевидно, что выводы, полученные на различных непересекающихся выборках, могут значительно отличаться друг от друга. Сопоставление результатов исследований на различных выборках объективно очень затруднено.

Более того, работы, анализирующие полную выборку сделок слияний, значительно отличаются по рассматриваемому периоду. В среднем исследователи рассматривают одно десятилетие. Однако различные волны слияний в XX веке отличаются качественно и структурно, что в свою очередь также не предполагает полного соответствия полученных выводов.

С другой стороны, значительные различия коренятся в методах анализа. Аномальная доходность может определяться относительно исторической доходности акций, рынка или компаний-аналогов.

Существенные различия научных работ заложены в исследуемом временном отрезке динамики акций. Несмотря на то что большинство работ отталкиваются от даты первого сообщения о сделке, существует целый комплекс работ, анализирующих доходность относительно даты закрытия сделки. Так, Сервес [Servaes, 1991] рассматривает динамики акций в момент окончательного закрытия сделки, а Лугран и Вийх [Loughran\&Vijh, 1997] спустя 5 лет. Хотя, как правило, исследователи рассматривают временное окно вокруг появления первого сообщения, этот диапазон также существенно разнится: от одного дня Мулерейн и Бунн [Mulherin\&Boone, 2000], недели - Каплан и Вайсбах [Kaplan\&Weisbach, 1992] и двух месяцев до и через два месяца после сделки - Макиейра [Maquieira, 1998].

Итак, можно сделать вывод, что, несмотря на простоту метода, результаты, полученные исследователями, существенно отличаются друг от друга ввиду различий анализируемых выборок, бесконечных вариаций метода и рассматриваемых временных периодов.

Метод анализа финансовых показателей. В данном подходе изучается публикуемая финансовая отчетность компаний до и после слияния. В результате анализируется, как изменяются финансовые показатели. В различных работах акцентируется внимание на различных аспектах: чистая прибыль, доходность активов, прибыль на акцию, операционный рычаг или ликвидность фирмы.

Как правило, в подобных работах рассматриваются сходные компании отрасли, которые, с одной стороны, пережили слияния и, с другой, у которых подобного опыта не было. Это позволяет сравнить влияние слияний и поглощений не результативность функционирования компании.

Преимуществом данного метода можно назвать определенную достоверность данных. Ведь отчетность компаний, как правило, должна быть проверена аудиторами, что теоретически должно исключать возможные махинации. В реальности это, конечно, не всегда так. 
Также, поскольку отчетность компаний используются инвесторами для оценки компаний, по изменению в финансовой отчетности можно косвенно оценить создаваемую добавленную стоимость от сделки.

С другой стороны, финансовая отчетность предполагает ряд существенных недостатков. Во-первых, бывают случаи, когда финансовые отчеты разных годов даже одной компании невозможно сравнить между собой, поскольку фирма может сменить свою политику бухгалтерского учета. К тому же сами стандарты учета очень часто изменяются.

Во-вторых, важным недостатком использования финансовой отчетности является то, что она учитывает лишь историческую информацию, а будущие возможности, открываемые сделками слияний и поглощений, остаются за рамками анализа.

В-третьих, в рамках бухучета очень сложно решается проблема корректной оценки нематериальных активов. С учетом того, что эта часть баланса с каждым годом набирает все большую значимость, это накладывает очень большие ограничения на анализ с помощью данного подхода. Так, высокотехнологичные отрасли практически не рассматриваются.

В-четвертых, результаты очень чувствительны к инфляции, поскольку бухгалтерские статьи не всегда адекватно корректируются в связи с изменениями макроэкономических параметров.

В-пятых, данный метод относительно негибкий, поскольку между различным датами отчетности проходят очень большие промежутки времени .

Наконец, различия в стандартах бухгалтерской отчетности между различными странами или даже компаниями очень усложняют анализ международных сделок слияний и поглощений.

Данные исследования можно условно разделить на анализ доходности для владельцев акций поглощаемой компании (далее называемой жертвой) и компании-поглотителя (далее называемой покупателем).

В целом доходности распределены равномерно. В 1/3 случаев наблюдается отрицательная доходность, в $1 / 3$ практически изменений не происходит, и еще в $1 / 3$ отмечается значимая положительная доходность.

Исследования показывают, что прослеживается тенденция уменьшения доходности с течением времени. Так, наблюдаемая доходность в 60-70-е годы превосходит результативность 90-х. Исключением является банковская сфера, где доходность, напротив, возросла.

Если смотреть совокупную доходность всех акционеров, то она получается в большинстве случаев положительной.

Наблюдающиеся различия в выводах также могут быть объяснены существенным отличием предмета и методов анализа финансовых данных. Можно предположить, что использование показателей доходности ROA Микс [Meeks, 1977], ROE Сальтер [Salter, 1979], ROC Херман, Ловенштайн [Herman, Lowenstein, 1988] или валовой маржи Шарма [Sharma, 2002] могут приводить к другим результатам, нежели анализ рыночной доли Мюллер [Mueller, 1985] или оборачиваемости капитала Хейли, Палперу и Рубак [Неaly, Palepu\&Ruback, 1992].

Наряду с непосредственным анализом финансовой отчетности множество исследователей значительно корректируют исходные данные. Так, в работе Паррино, Харрис [Parrino, Harris, 2001] рассматриваются скорректированные операционные денежные потоки, a Cет [Seth, 1990] анализириует потоки для акционеров.

Далее значительны различия видны в мощности выборки: от 16 сделок Сальтер и Вейнхолд [Salter\&Weinhold, 1979] до 613 у Дикерсон, Гибсон Тсакалотос [Dickerson, Gibson, Tsakalotos, 1997]; анализируемые страны: от Великобритании — Микс [Meeks, 1977] до Австралии - Шарма [Sharma, 2002]; временные периоды: от 60-х у Сальтер [Salter, 1979] до XXI века - Хо [Но, 2006]; исследуемый диапазон: от года — Равеншафт [Ravenscraft, 1987] до 5 лет - Дикерсон [Dickerson, 1997]. 
Таким образом, так же как и в случае с анализом котировок акций, различие в выводах обеспечивается различием в используемом инструментарии, выборке и временном периоде.

Метод опросов Брюнера [Bruner, 2004]. В данном подходе напрямую опрашиваются управляющие компаний, переживших слияния. В опросе исследователи пытаются узнать, какую добавленную стоимость принесли M\&A для компании. Стандартизированные вопросы позволяют на основе имеющейся выборки вывести определенные закономерности для всей совокупности сделок.

Преимущество данного подхода заключается в том, что некоторые скрытые аспекты, создающие добавленную стоимость в результате слияний и поглощений, могут в открытую быть незамеченными на фондовом рынке. Инсайдерская информация может значительно обогатить анализ сделок.

С другой стороны, в этой сфере могут открыться агентские проблемы. Некоторые менеджеры, не являющиеся акционерами, могут преследовать совсем другие цели, нежели собственники. Поэтому оценка менеджерами экономической добавленной стоимости, создаваемой при слиянии и поглощении, может оказаться очень субъективной.

К тому же некоторые не совсем хорошие результаты в опросах могут быть менеджерами представлены в более выгодном свете. А некоторые компрометирующие факты попросту могут быть опущены.

У иных исследователей также вызывает критику количество опрашиваемых менеджеров. Недостаточное их количество делает обобщение результатов на всю генеральную совокупность сделок необоснованным.

Данные работы, по большому счету, выполняются практиками, которые многие годы работают в сфере слияний и поглощений. В данных исследованиях выводы, как правило, формируются на основе непосредственного опроса небольшого количества управляющих.

Очень часто подобные работы для лучшего их понимания выполняются не в строгом научном стиле, а в более свободном формате. Это конечно же значительно снижает возможность использования данных исследований в более серьезных разработках.

Тем не менее результаты опросов очень схожие, характерные и для других подходов. Шесть исследований из 13 отражают отрицательные результаты, а остальные — либо положительный, либо отрицательный итог.

Таблица 1.

Наиболее интересные исследования, основанные на опросах управляющих

\begin{tabular}{|c|c|c|c|}
\hline $\begin{array}{c}\text { Источник и } \\
\text { дата }\end{array}$ & Размер выборки & Период & Результаты \\
\hline $\begin{array}{l}\text { Johan Brjoksten } \\
, 1965\end{array}$ & $\begin{array}{l}5409 \\
\text { промышл } \\
\text { слияний } \\
\end{array}$ & 195 & \begin{tabular}{|llr} 
В $16 \%$ случаев & наблюдаются неудачи \\
финансовые, & стратегические \\
технологические & & \\
\end{tabular} \\
\hline PA Consulting & $\begin{array}{l}28 \text { гл } \\
\text { В банг }\end{array}$ & 1982 & $\begin{array}{l}80 \% \text { поглощений негативно влияют на } \\
\text { цену акций компании- поглотителя }\end{array}$ \\
\hline $\begin{array}{l}\text { David Mitchell } \\
\text { of Economists } \\
\text { Intelligence } \\
\text { Unit, } 1996 \\
\end{array}$ & $\begin{array}{l}\text { Исследование } \\
\text { управляющих в } 150 \\
\text { компаниях }\end{array}$ & $1992-$ & \begin{tabular}{|l}
$30 \%$ называют произошедшее слияние \\
удачным, желательным для повторения; \\
$53 \% \quad$ - удовлетворительным, но \\
нежелательным для повторения; 11\% - \\
неудачным; 5\% - разрушительным \\
\end{tabular} \\
\hline $\begin{array}{l}\text { Michael Mayo, } \\
\text { ehman Bros. }\end{array}$ & О Оанков & & $\begin{array}{l}\text { Акции поглотителя падают в среднем на } \\
10 \% \text { за неделю после опубликования } \\
\text { информации, но в течение } 2 \text { месяцев } \\
\text { быстро восстанавливается }\end{array}$ \\
\hline
\end{tabular}




\begin{tabular}{|l|l|l|l|}
\hline $\begin{array}{l}\text { Andersen } \\
\text { Consulting }\end{array}$ & Большие сделки & 1994-1997 & $\begin{array}{l}44 \% \text { всех сделок не оправдало } \\
\text { финансовых и стратегических ожиданий }\end{array}$ \\
\hline $\begin{array}{l}\text { Chaudhuri and } \\
\text { Tabrizi (1999) }\end{array}$ & $\begin{array}{l}\text { поглощения 24 } \\
\text { high-tech компаний }\end{array}$ & $\begin{array}{l}\text { бтоли оценены положительно обоими } \\
\text { показали нулевую отдачу от инвестиций }\end{array}$ \\
\hline
\end{tabular}

Источник: Bruner R. Does M\&A Pay? // Journal of Financial Economics, 2004.

Метод кейсов Брюнера [Bruner, 2004]. В данных работах рассматриваются либо конкретные сделки, либо их очень ограниченная выборка. В них очень глубоко и детально анализируются конкретные случаи. Как правило, исследователи прибегают к подробному интервью с участниками и заинтересованными сторонами. Зачастую исследования основаны на инсайдерской информации. Данный подход позволяет выявить какие-то новые закономерности, которые впоследствии проверяются другими подходами.

Также преимуществом данного подхода можно считать то, что каждая конкретная сделка изучается со всех сторон, что позволяет добиться объективности результатов.

С другой стороны, результаты одной конкретной сделки не позволяют утверждать, что выводы характерны для всех сделок. Очень значим фактор случайности.

Ввиду детального анализа сделок и изучения инсайдерской информации количество подобных исследований в общей массе работ, фокусирующихся на теме слияния и поглощения, не велико.

Лис и Винсент [Lys\&Vincent, 1995] рассматривали, как AT\&T поглотила NCR Corporation. Эта сделка уменьшила богатство акционеров AT\&T от \$3,9 до \$6,5 млрд. Автор предложил несколько вариантов объяснения этого факта. Во-первых, в сделке явно прослеживаются мотивы менеджеров, противоречащие интересам акционеров. Во-вторых, наблюдается излишняя самоуверенность менеджеров и опора на собственное субъективное видение вопреки объективным фактам.

Брюнер [Bruner, 1999] изучил неудавшуюся попытку слияния AB Volvo и Renault в 1993 году. Появление информации о готовящейся сделке понизило котировки Volvo на 22\% до тех пор, пока совет директоров компании не отказался от своего намерения. Данное исследование показывает: снижение стоимости компании было связано с тем, что рынок не поверил в возможную синергию и возможность получения контроля над Renault.

Брюнер и Идс [Bruner\&Eades, 1992] и Врюк [Wruck, 1991] проанализировали банкротство Revco Drug Stores, менеджеры которой провели крупнейшее в отрасли MBO (выкуп компании за счет заемных средств). Неудача, как выяснили авторы, была связана с высокой ценой покупки, агрессивным использованием долга, вызванными излишней самоуверенностью менеджмента.

Калпан, Митчел и Врюк [Kaplan, Mitchell\&Wruck, 1997] рассмотрели две сделки, при появлении информации о которых котировки компаний-покупателей вели различным способом (в одном случае негативная, в другом - позитивная). Как выяснилось позже на интерьвю, ни одна из сделок не увенчалась успехом. Менеджеры объясняют свою неудачу недостатком информации о покупаемой компании и неоптимальной структурой сделки.

Хиетала, Каплан и Робинсон [Hietala, Kaplan\&Robinson, 2002] рассмотрели покупку Paramount компанией Viacom. В своем анализе авторы вычленили в динамике акций компании величину изменения, обеспеченную эффектом синергии, и величину изменения, вызванную очень высокой ценой покупки. Исследователи рассчитали, что Viacom переплатил более \$2 млрд, несмотря на то что глава Viacom владеет $3 / 4$ своей компании.

Рюбак [Ruback, 1982] предположил, что чистый прирост стоимости создается совместно для акционеров обеих компаний. Так, сумма стоимости, созданной для акционеров компании-цели Conoco (3,2 млрд), и стоимости, потерянной акционерамипокупателями (DuPont) (\$0,8 млрд), в итоге дают совокупный выигрыш в размере \$2,4 млрд. Рюбак предложил несколько вариантов возможных причин роста и потери стоимости для акционеров, но затруднился с объяснением причин анализируемой сделки. 
Обзор существующих исследований показывает, что проблема слияний и поглощений широко изучалась множеством авторов в течение длительного промежутка времени в различных регионах мира.

Парадоксально, но ни одна из гипотез относительно закономерностей слияний и поглощений, их влияния на благосостояние акционеров до сих пор не доказана с достаточной степенью надежности. Для каждого суждения можно найти достаточное количество работ, доказывающих противоположные утверждения.

Тем не менее в научной среде, в отличие от бизнеса, неоправданно сложилась искаженная картина процессов слияний и поглощений:

1. После появления информации о сделке стоимость акций приобретаемой компании растет.

2. После появления информации о сделке стоимость акций компании-покупателя падает.

3. После сделки финансовые показатели компаний падают.

Мы считаем, что данная картина значительно искажена, поскольку существует множество научных и практических свидетельств обратного. Мы считаем, что это вызвано следующими факторами:

1. Неудачные результаты сделок слияния и поглощения имеют больший резонанс.

2. Не все научные работы широко известны.

3. Ограниченность (ошибочность) предпосылок и ограниченность выборки научных работ.

Данное устоявшееся видение интеграционных процессов требует переосмысления, поскольку не согласуется со множеством новых данных, в частности о сделках на развивающихся рынках.

Дальнейшее изучение данного вопроса требует обобщающих работ с ясными предпосылками и на большой выборке данных.

Несмотря на существование множества разных стимулов к слияниям и поглощениям компаний, мы предполагаем, что рынок капитала способен оценивать экономические мотивы и вычислять те, что обеспечивают непосредственный рост финансовых показателей. Мы предполагаем, что средний временной горизонт участников рынка невелик и ограничивается ближайшими 3-4 годами. Поэтому стратегические сделки, нацеленные на более отдаленный результат, адекватно рынком не оцениваются.

Таким образом, мы считаем, что в момент появления информации о сделке величина наблюдающейся избыточной, или аномальной, доходности (abnormal return), которая измеряется как разность доходности акций изучаемой компании и эталонной, нормальной доходности, определяется рынком на основе независимой оценки инвесторами целесообразности и эффективности предстоящей сделки. Данная гипотеза очень тесно связана с гипотезой эффективного рынка, которая предполагает, что рынок быстро и эффективно учитывает в котировках ценных бумаг компании всю доступную информацию. Таким образом, подтверждение одной гипотезы влечет за собой соответствующее подтверждение другой. В данной работе предварительное тестирование гипотезы эффективности самого рынка не проводилось, и мы предполагаем, что исследуемые рынки отвечают критериям эффективности.

\section{I. Модель исследования на основе взаимосвязи перспективных и} ретроспективных показателей

В данной работе реализован новый подход к оценке сделок слияний/поглощений, сочетающий ретроспективный и перспективный анализ. Мы применяем одновременный анализ показателей финансовой отчетности компаний до и после сделки слияния/поглощения и динамики их котировок в момент поступления информации о предстоящей интеграции. 
Анализ финансовой отчетности предполагает сравнение показателей эффективности отдельных бизнесов до интеграции с аналогичными показателями интегрированных компаний двумя годами позже. В данной работе в качестве бухгалтерского показателя эффективности мы применяем рентабельность, рассчитанную на основе показателя прибыли до вычета процентов, налогов и амортизации (EBITDA), поскольку эта форма прибыли более тесно связана с потоками денежных средств бизнеса и не искажена финансовой политикой компании. Для отражения перспективного ракурса анализа мы рассматриваем динамику котировок компаний в промежутке за неделю до и после первого сообщения о сделке. Доходность акций компаний-участниц сделки сравнивается с эталонной, и в качестве эталона использован рыночный индекс. Корректировка на рыночный индекс позволяет определить индивидуальную доходность ценных бумаг компании и выявить избыточную, или аномальную, доходность.

Имея две характеристики, отражающие, с одной стороны, ожидания инвесторов и реакцию рынка на новость о слиянии и финансовый результат интеграции двумя годами позже - с другой, мы определяем взаимосвязь между ними посредством корреляционного анализа. Для поиска взаимосвязи между реакцией рынка в момент появления информации о сделке и финансовыми результатами совместной компании мы используем регрессионный анализ. По нашему мнению, данный подход с достаточной степенью достоверности позволяет определить оценку рынком эффективности сделок слияний и поглощений. Мы исходим из гипотезы о том, что динамика котировок компании, улавливая ожидания, отражает оценку инвесторами экономической целесообразности сделки слияния/поглощения.

Реакция рынка на появление информации о конкретной сделке определяется относительно динамики рынка в целом. Под реакцией рынка в данной работе мы предполагаем доходность акций компаний во временном окне за неделю до первого сообщения и неделю спустя.

$$
r_{\text {aquirer } / \mathrm{t} \arg \mathrm{et}}=\frac{P_{- \text {week }}-P_{+ \text {week }}}{P_{- \text {week }}}
$$

где:

$\mathrm{r}_{\text {acuirer/target }}$ - реакция рынка на сделку;

$\mathrm{P}_{\text {-week }}$ - цена за неделю до первого сообщения о сделке;

$\mathrm{P}_{+ \text {week }}$ - цена неделю спустя после первого сообщения о сделке.

Мы предполагаем, что неделя - это достаточный горизонт для адекватного выделения эффекта информации о сделке. Недельный промежуток до официального появления информации о сделке необходим для учета инсайдерской информации, которая просочилась до того, как о будущей сделке узнала большая часть инвестиционного сообщества. С другой стороны, неделя после сообщения необходима для того, чтобы в котировках полностью отразилась вся полученная информация. Мы предполагаем, что необходимо определенное время, чтобы информация была получена всеми участниками рынка, адекватно оценена и осмыслена. Бесспорно, временной горизонт должен был достаточен, чтобы нивелировать первую эмоциональную реакцию участников рынка на появление информации и отражать лишь их взвешенные решения.

Индивидуальная доходность акций компаний корректируется в соответствии с доходностью рынка, которая выполняет роль эталонного показателя. Для этих целей используется доходность основного индекса биржи, на котором торгуются ценные бумаги компании.

$$
r_{\text {market index }}=\frac{I_{- \text {week }}-I_{+ \text {week }}}{I_{- \text {week }}},
$$


где:

$\mathrm{r}_{\text {marketindex }}$ - доходность рынка;

$\mathrm{I}_{\text {-week }}$ - индекс за неделю до первого сообщения о сделке;

$\mathrm{I}_{+ \text {week }}$ - индекс неделю спустя после первого сообщения о сделке.

Для измерения взаимосвязей между перспективными и ретроспективными показателями использована избыточная (аномальная) доходность акций компаний, которая рассчитана следующим образом:

(3) $r_{\text {anormal }}=r_{\text {target/aquirer }}-r_{\text {market index }}$,

где:

$\mathrm{r}_{\text {anormal }}$ - аномальная доходность;

$\mathrm{r}_{\text {target/acquirer }}-$ доходность акций компании;

$\mathrm{r}_{\text {marketindex }}$ - доходность рынка.

Мы полагаем, что данная операция позволяет скорректировать показатель доходности акций компаний-участниц сделки с динамикой всего рынка и избежать влияния общеэкономических факторов.

В данной работе мы используем расчеты аномальной доходности акций только поглощающей компании. Мы считаем, что исследование динамики котировок компаниицели может быть значительно искажено краткосрочными факторами, не связанными с эффективностью сделки. Во-первых, с одной стороны, сделки слияния совершаются по цене, близкой к справедливой, тем самым создается ценовой ориентир для инвестиционного сообщества. С другой стороны, большинство акций торгуется с определенным дисконтом к справедливой из-за ряда факторов, в частности из-за низкой ликвидности. В большинстве случаев это приводит к тому, что ценовой ориентир, близкий к справедливой стоимости, и временное снижение роли фактора низкой ликвидности объективно приводят к тому, что котировки акций компании-цели вырастают при появлении информации о сделке. Мы считаем, что объективно оценить факторы роста котировок поглощаемой компании, вызванные только долгосрочными экономическими выгодами от слияния, практически невозможно.

Таким образом, оценка котировок акций компании-цели остается за рамками данной работы. Анализ фокусируется на аномальной доходности акций поглощающей компании.

В качестве ретроспективного показателя в данной работе мы используем динамику рентабельности продаж на основе EBITDA. В данной работе, имея данные по выручке и EBITDA отдельных компаний до слияния, мы оцениваем синтетическую рентабельность, как если бы компании уже работали вместе (формула 4). При этом сделано допущение о том, что показатели прибыли и выручки компаний-участниц сделки не ухудшатся после сделки.

(4) EBITDAm $\arg$ in $_{\text {synthetic }}=\frac{E B I T D A_{t_{\arg e t}}+E B I T D A_{\text {aquirer }}}{\text { Sales }_{t_{\text {arg } e t}}+\text { Sales }_{\text {aquirer }}}$,

где:

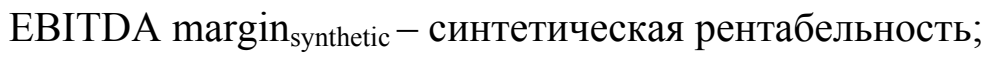

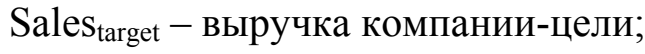

EBITDA $_{\text {target }}$ - EBITDA компании-цели;

Sales acquirer - выручка компании-покупателя;

EBITDA $_{\text {acquirer }}$ - EBITDA компании-покупателя.

Для определения реального результата слияния был исследован период протяженностью два года после сделки, и он измеряется через бухгалтерский показатель рентабельности продаж по данным годовой отчетности, в которой консолидированы финансовые результаты обеих компаний, на основе формулы 5: 
(5) EBITDA $m \arg$ in $_{+2 \text { year }}=\frac{E B I T D A_{\text {consoliadted }}}{\text { Sales }_{\text {consolidated }}}$,

где:

EBITDA margin $_{+2}$ years - рентабельность по EBITDA два годя спустя;

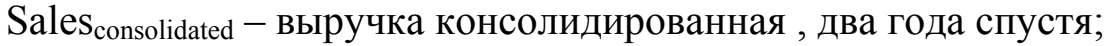

EBITDA $_{\text {consolidated }}$ - EBITDA консолидированная , два года спустя.

Показатель для измерения чистого эффекта сконструирован следующим образом. Была рассчитана разница между рентабельностью спустя два года и синтетической рентабельностью до слияния (формула 6):

(6) Net $m$ arg in effect $=E B I T D A_{+2 \text { year }}-E B I T D A_{\text {synthetic }}$,

где:

Net margin effect - чистый эффект слияния;

EBITDA margin $_{+2}$ years - рентабельность по EBITDA два годя спустя;

EBITDA margin synthetic $_{-}$синтетическая рентабельность.

Имея два массива данных - бухгалтерские показатели эффекта и финансовые, рыночные показатели эффекта, - мы выявляем взаимосвязь между ними путем проведения регрессионного анализа. Коэффициент детерминации $\left(\mathrm{R}^{\wedge} 2\right)$ полученной регрессии является показателем силы взаимосвязи между данными. Значительная взаимосвязь подтверждает гипотезу об эффективной оценке рынком сделок слияний/поглощений.

Бесспорно, предложенный метод обладает рядом недостатков, которые могут искажать полученные результаты.

Во-первых, относительно широкое двухнедельное временное окно при оценке аномальной доходности предполагает учет не только эффекта слияния, но и других новостей, которые могут отразиться на динамике котировок компании.

Во-вторых, финансовые результаты консолидированной компании вычисляются из ближайшей, второй после сделки, годовой отчетности. Поскольку временной промежуток между фактической сделкой и годовой отчетностью значительно разнится, то в анализируемой выборке данных периоды совместных операций интегрированных компаний отличаются друг от друга, что предполагает некую неоднородность выборки.

В-третьих, для некоторых отраслей двухгодичный промежуток является незначительным для реализации эффекта от слияния. Однако увеличение временного горизонта создает риск того, что рост финансовых показателей будет вызван не эффектом слияния, а иными другими причинами.

В-четвертых, метод не учитывает отраслевых тенденций, которые могут значительно отличаться от экономики в целом. Данный недостаток может быть устранен посредством сравнения с компаниями-аналогами.

В-пятых, подход не позволяет количественно измерить стратегические или какие-либо долгосрочные параметры, достижение которых являлось целью слияния/поглощения.

\section{II. Построение и анализ выборки}

В данной работе анализ полностью опирается на данные, полученные из информационных систем Bloomberg, Thompson OneBanker и MergerMarket. Изначально отбиралась выборка всех сделок, удовлетворяющих следующим параметрам:

1. Сделка была совершена в период с 2001-го по 2004 год.

Выборка была построена начиная с 2001 года, поскольку информационные базы не имеют удовлетворительной статистики по более поздним сделкам. Во-вторых, мы считаем, что в этот период полученные данные являются довольно объективной базой для исследования, поскольку период характеризуется отсутствием каких-либо пузырей.

2. Размер сделки превышает \$200 млн. 
Мы считаем, что сделка должна быть существенной, заметной на рынке.

3. Покупаемая доля превышает $75 \%$.

В работе мы предполагаем, что приобретение контроля над компанией обеспечивает активное ее преобразование, интеграцию в поглощающую компанию. Полный контроль над бизнесом обеспечивает реализацию всех преимуществ и синергий от слияния.

4. Поглощающая компания является публичной, и ее акции котируются.

Высокая котировка компании является необходимой для расчета аномальной доходности при появлении информации о сделке. Публичность поглощаемой компании не является необходимой, поскольку в рамках данной работы доходность для компании цели не вычисляется ввиду наличия очевидных недостатков.

Таблица 2.

Краткая характеристика используемых данных, тыс. долл.

\begin{tabular}{|l|l|l|l|l|}
\hline Количество наблюдений & 317 & & & \\
\hline Дата & $2001-2004$ & & & \\
\hline & Максимум & Среднее & Медиана & Минимум \\
\hline Размер сделки & 64264 & 2079 & 718 & 200 \\
\hline Выручка компании-цели & 331170 & 4887 & 675 & 4 \\
\hline ЕВITDA компании-цели & 31437 & 690 & 94 & -108 \\
\hline Средняя маржа по ЕВITDA & $\mathrm{n} / \mathrm{m}$ & $17 \%$ & $15 \%$ & $\mathrm{n} / \mathrm{m}$ \\
\hline Выручка компании-покупателя & 942356 & 40786 & 11155 & 147 \\
\hline ЕВITDA компании-покупателя & 212428 & 7888 & 1970 & -1988 \\
\hline $\begin{array}{l}\text { Средняя рентабельность по } \\
\text { ЕВITDА }\end{array}$ & $\mathrm{n} / \mathrm{m}$ & $24 \%$ & $20 \%$ & $\mathrm{n} / \mathrm{m}$ \\
\hline
\end{tabular}

Для каждой компании из полученной выборки из соответствующей годовой отчетности определяется выручка и EBITDA. Для поглощающей компании определялись финансовые показатели как до сделки, так и через два года после нее, содержащие результат уже двух интегрированных компаний.

Ввиду закрытости многих частных компаний величина EBITDA остается непрозрачной, поэтому сделки с неполными финансовыми данными были исключены из выборки.

Информационные базы предоставляют данные по дате появления первой информации о сделке. Отталкиваясь от этой даты из базы сделок, предоставленной информационными системами, были получены котировки акций поглощающей компаний за неделю до и через неделю после первого сообщения о сделке. Параллельно были получены соответствующие данные по значениям индексов бирж, на которых торгуются акции поглощающих компаний. 


\section{Использованные национальные индексы}

\begin{tabular}{|l|l|l|l|}
\hline Индекс & Сокращение & Страна & Тип рынка \\
\hline China Shanghai Comp & SSEC & Китай & Развивающийяя \\
\hline Finland Helsinki General & HEX & Финляндия & Развитой \\
\hline Israel TA-100 & TA100 & Израиль & Развивающийся \\
\hline Australia All Ordinaries & ASX & Австралия & Развитой \\
\hline AMEX Composite & XAX & США & Развитой \\
\hline Germany DAX & DAX & Германия & Развитой \\
\hline Spain Madrid General & IGBM & Испания & Развитой \\
\hline Austria ATX & ATX & Австрия & Развитой \\
\hline Brazil Bovespa & BUSP & Бразилия & Развивающийся \\
\hline Italy MIBTel & MIBTEL & Италия & Развитой \\
\hline Canada TSE 300 Comp & TSE & Канада & Развитой \\
\hline France CAC 40 & CAC & Франция & Развитой \\
\hline UK FTSE 100 & FTSE & Британия & Развитой \\
\hline Russian RTS index & RTSI & Россия & Развивающийся \\
\hline Argentina MerVal & MERVAL & Аргентина & Развивающийся \\
\hline Japan Nikkei 225 & NIKKEI & Япония & Развитой \\
\hline S\&P 500 Index & SPX & США & Развитой \\
\hline Hong Kong Hang Seng & HSI & Гонконг & Развитой \\
\hline
\end{tabular}

По результатам расчетов анализируемых показателей из выборки были исключены компании с аномально высокими/низкими показателями.

Исходная выборка для последующего анализа была разделена на следующие подгруппы:

1. География (компания-покупатель):

a) развитые страны,

б) развивающиеся страны.

2. Размер компании:

a) крупные компании (выручка свыше $\$ 10$ млрд),

б) маленькие компании (выручка менее $\$ 10$ млрд).

Таблица 4.

Краткая характеристика выборки данных, тыс. долл.

\begin{tabular}{|l|l|l|l|l|}
\hline & Крупные & Остальные & Развитые & Развивающиеся \\
\hline Количество наблюдений & 168 & 149 & 277 & 40 \\
\hline $\begin{array}{l}\text { Средняя выручка } \\
\text { компании-цели }\end{array}$ & 73338 & 4082 & 30109 & 114720 \\
\hline ЕВITDА компании-цели & 13992 & 1006 & 5822 & 22198 \\
\hline $\begin{array}{l}\text { Средняя рентабельность } \\
\text { по ЕВITDA }\end{array}$ & $21 \%$ & $28 \%$ & $24 \%$ & $24 \%$ \\
\hline
\end{tabular}

\section{III. Переменные модели и их оценка}

Подсчет показателей эффективности разделяется на вычисление ретроспективных и перспективных показателей. На основе данных годовой финансовой отчетности были вычислены показатели рентабельности по EBITDA для каждой компании, участвовавшей в сделке. На основе исторических данных путем взвешивания по выручке были сформированы 
синтетические рентабельности объединяющихся компаний, как если бы они были интегрированы годом ранее.

Таблица 5.

Дескриптивная статистика рентабельности по EBITDA

\begin{tabular}{|l|l|l|l|}
\hline \multicolumn{1}{|c|}{$\begin{array}{c}\text { Фактическая } \\
\text { рентабельность }\end{array}$} & Компания-цель & $\begin{array}{c}\text { Компания- } \\
\text { покупатель }\end{array}$ & $\begin{array}{c}\text { Синтетическая } \\
\text { рентабельность }\end{array}$ \\
\hline Среднее & $17 \%$ & $24 \%$ & $24 \%$ \\
\hline Медиана & $15 \%$ & $20 \%$ & $20 \%$ \\
\hline $\begin{array}{l}\text { Стандартное } \\
\text { отклонение }\end{array}$ & $33 \%$ & $18 \%$ & $18 \%$ \\
\hline Эксцесс & 24,4 & 6,3 & 6,8 \\
\hline Кривизна & $-3,4$ & 0,1 & 0,1 \\
\hline
\end{tabular}

Далее на основе консолидированных финансовых показателей по состоянию через два года спустя сделки вычисляется изменение рентабельности.

Таблица 6.

\section{Дескриптивная статистика изменений рентабельности по ЕBITDA}

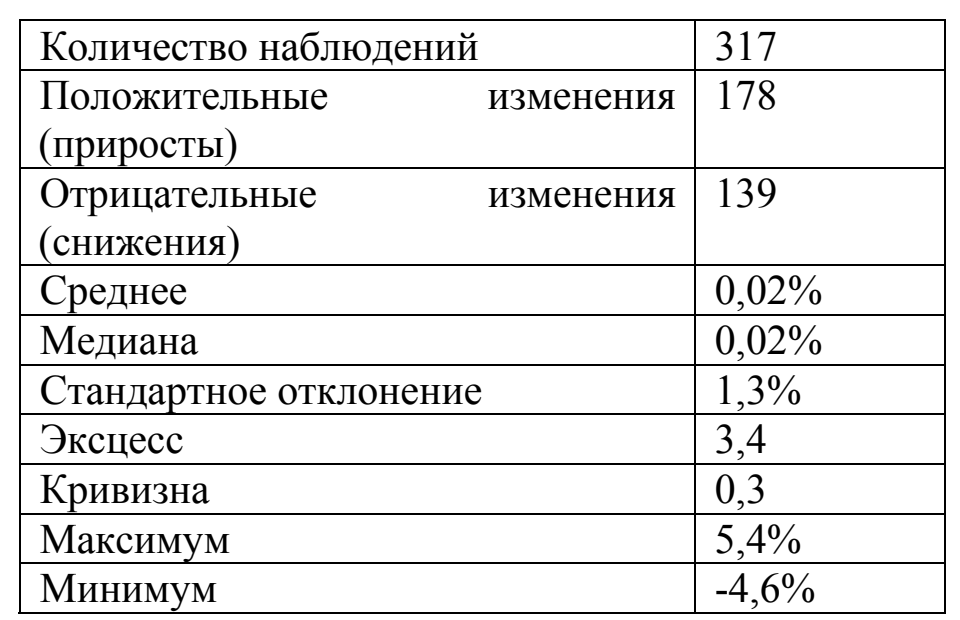

Таким образом, мы предполагаем, что рост эффективности бизнеса обеспечивается за счет синергетических эффектов сделки. Для наглядности статистические характеристики показателей изменения рентабельности представлены на рисунке 1. 


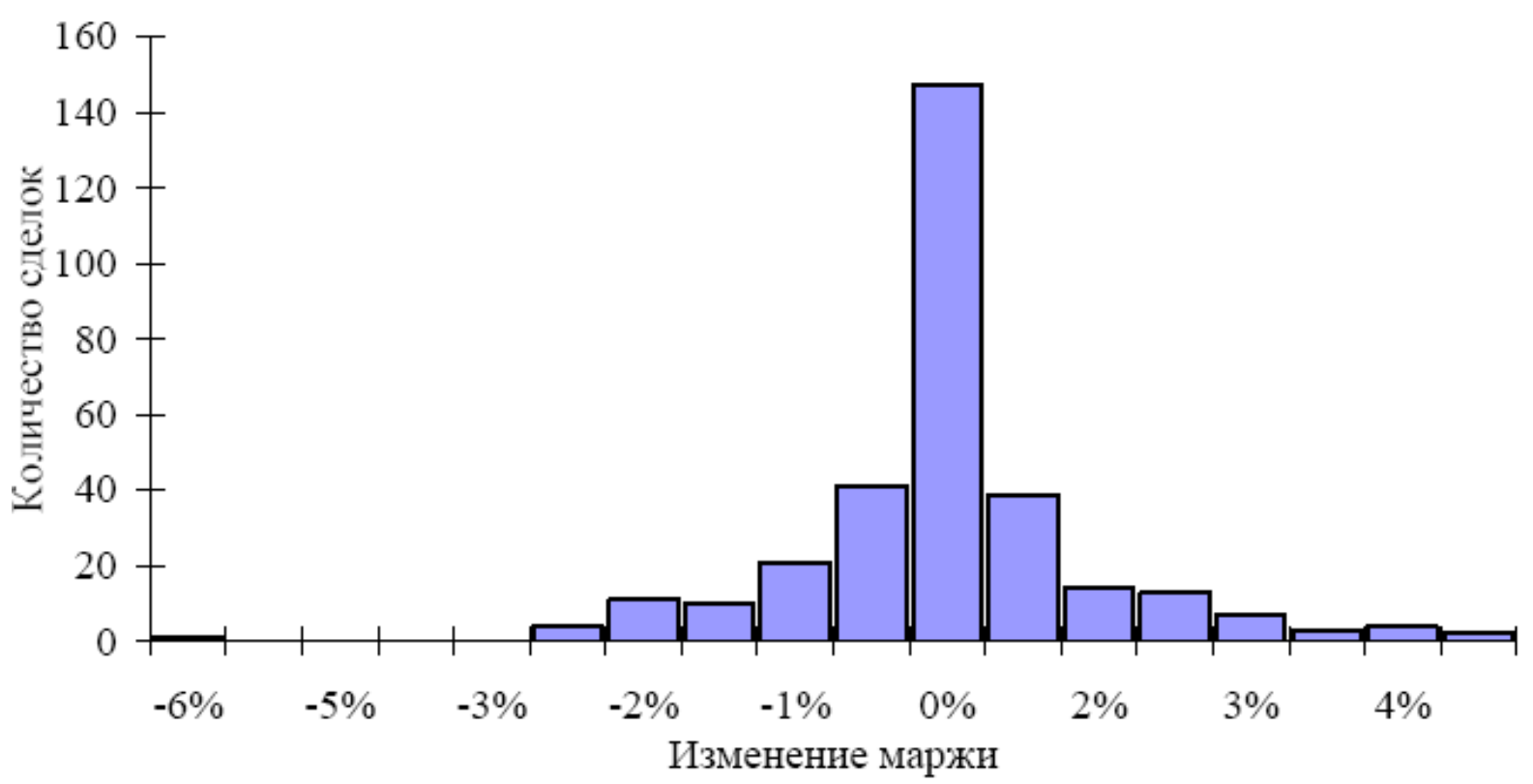

Рисунок 1. Гистограмма распределения изменения рентабельности по EBITDA

Мы считаем, что, базируясь на данных ретроспективного анализа, мы можем объективно судить об экономической целесообразности сделки и использовать эти данные для анализа математическим аппаратом.

Подсчет перспективных показателей отталкивается от данных котировок поглощающей компании за неделю до и через неделю после появления информации о сделке. Исходя из котировок компании вычисляется индивидуальная доходность для акций компании. Соответственно в этих же временных рамках вычисляется доходность отдельных национальных фондовых индексов. Разница между индивидуальной доходностью и доходностью фондовых индексов формирует аномальную доходность (таблица 7).

Таблица 7.

\section{Дескриптивная статистика аномальной доходности}

\begin{tabular}{|l|l|}
\hline Количество наблюдений & 317 \\
\hline$>0$ & 178 \\
\hline$<0$ & 139 \\
\hline Среднее & $0,03 \%$ \\
\hline Медиана & $0,03 \%$ \\
\hline Стандартное отклонение & $1,18 \%$ \\
\hline Эксцесс & 3,6 \\
\hline Кривизна & 0,2 \\
\hline Максимум & $4,6 \%$ \\
\hline Минимум & $-5,7 \%$ \\
\hline
\end{tabular}

На рисунке 2 приведена картина распределения показателей избыточной (аномальной) доходности. 


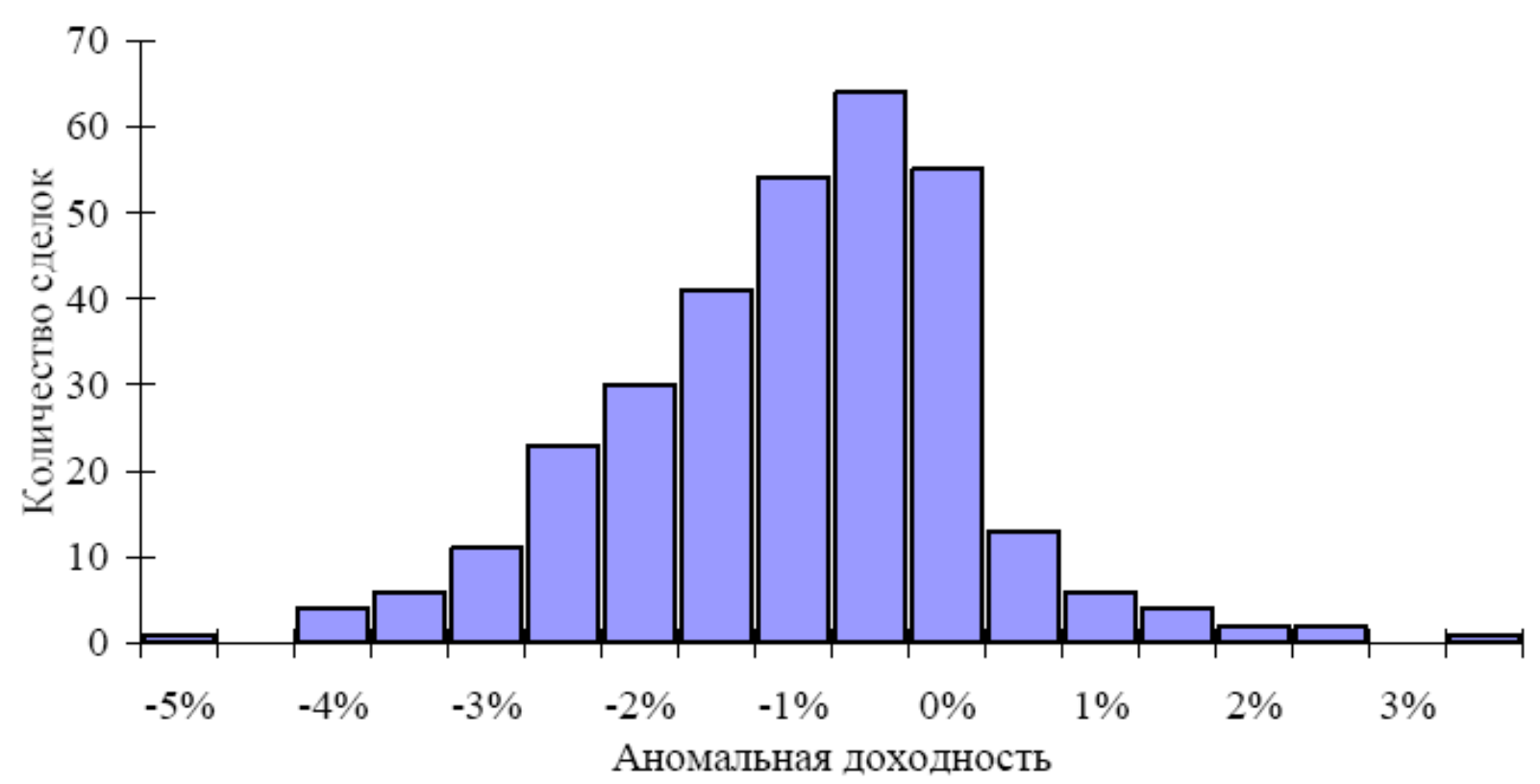

Рисунок 2. Гистограмма распределения аномальной доходности

Таким образом, при построении регрессий авторы оперируют следующими переменными: в качестве переменной X взято изменение рентабельности по EBITDA, в качестве переменной Y использована избыточная доходность.

IV. Описание и анализ результатов эмпирических тестов

Построение регрессионных связей в данной работе осуществляется с помощью математического аппарата электронных таблиц Microsoft Excel.

Анализ совокупной выборки

Гипотеза Н0: существует значимая зависимость между аномальной доходностью и изменением рентабельности по EBITDA.

Построение регрессии осуществляется по всей выборке, состоящей из 317 наблюдений. Результаты корреляционного анализа показаны на рисунке 3.

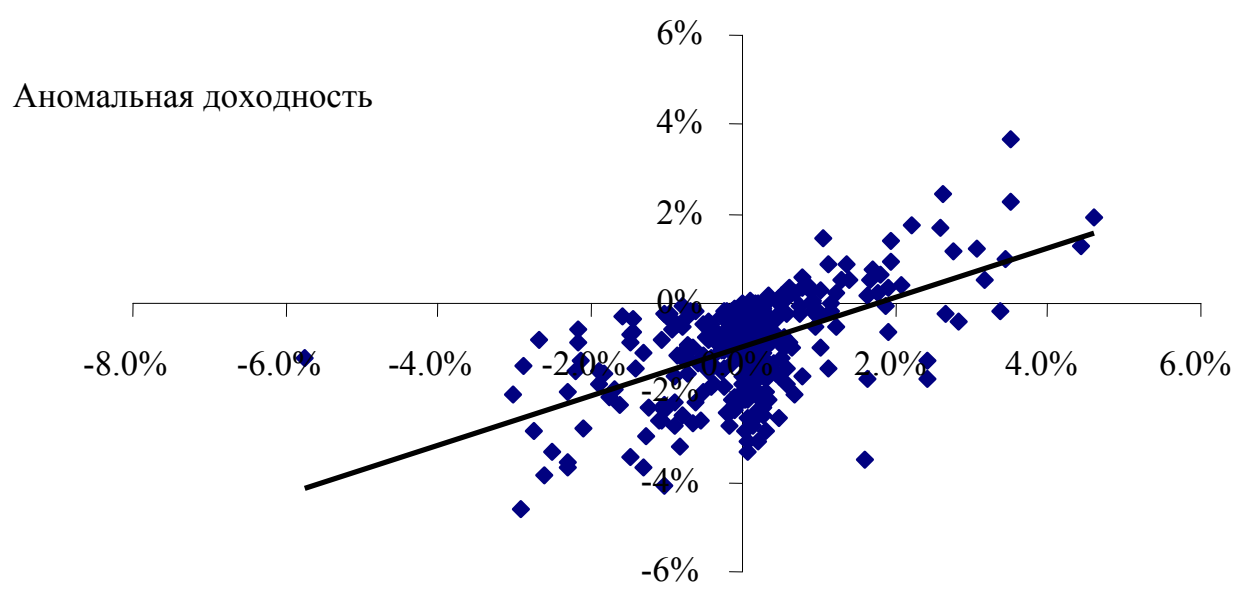

Рост рентабельности по EBITDA

Рисунок 3. Регрессия между аномальной доходностью иизменением рентабельности компании (полная выборка)

Корреляционный анализ показывает наличие сильной линейной взаимосвязи между реакцией рынка на сообщение о сделке и будущим ростом эффективности, вызванным 
влиянием интеграционной сделки. Коэффициент корреляции составляет 0,7. Результаты регрессии приведены в таблице 8.

Таблица 8.

Краткая характеристика регрессии между аномальной доходностью и изменением рентабельности компании

\begin{tabular}{|l|l|}
\hline $\mathrm{R}^{\wedge} 2$ & 0,39 \\
\hline Скорректированный $\mathrm{R}^{\wedge} 2$ & 0,36 \\
\hline Стандартная ошибка & 0,009 \\
\hline F-статистика & $\approx 0$ \\
\hline Формула & $\mathrm{y}=0,6 \mathrm{x}-0.5 \%$ \\
\hline
\end{tabular}

Тесты на значимость (F) и устойчивость показывают значимость и устойчивость полученной регрессии. Гипотеза Н0 подтверждается.

Построение регрессии показывает положительную взаимосвязь между двумя величинами. Экономический смысл в том, что при осуществлении сделки слияния/поглощения в случае отсутствия какого-либо экономического эффекта наблюдается отрицательная избыточная доходность. Приравнивая данную регрессию к $0(\mathrm{y}=0)$, имеем, что х принимает значение $0,8 \%$; это подразумевает, что рынок требует минимального роста рентабельности на $0,8 \%$, для того чтобы котировки акций не изменились относительно индекса. По нашему мнению, это связано с тем, что любая интеграционная сделка связана с определенными расходам для компаний и включает комиссии консультантам (в среднем на рынке $0,6 \%$ от суммы сделок), транзакционные издержки и т.п.

При значительном росте эффективности бизнеса от сделки наблюдается положительная избыточная доходность акций поглощающей компании.

Таким образом, данный факт, во-первых, подтверждает гипотезу о том, что рынок с определенной степенью точности определяет экономическую целесообразность сделки слияния/поглощения. Одновременно это подтверждает гипотезу эффективности фондового рынка, согласно которой, в котировках учитывается вся возможная информация.

Разное временное окно

В рамках данной работы мы провели анализ зависимости между показателем эффективности слияния и аномальной реакцией рынка на новость о слияния при различных временных окнах. В качестве дополнительной выборки было выбрано временное окно в диапазоне за день до сообщения и день спустя. Результаты этого этапа показаны на рисунке 4. 


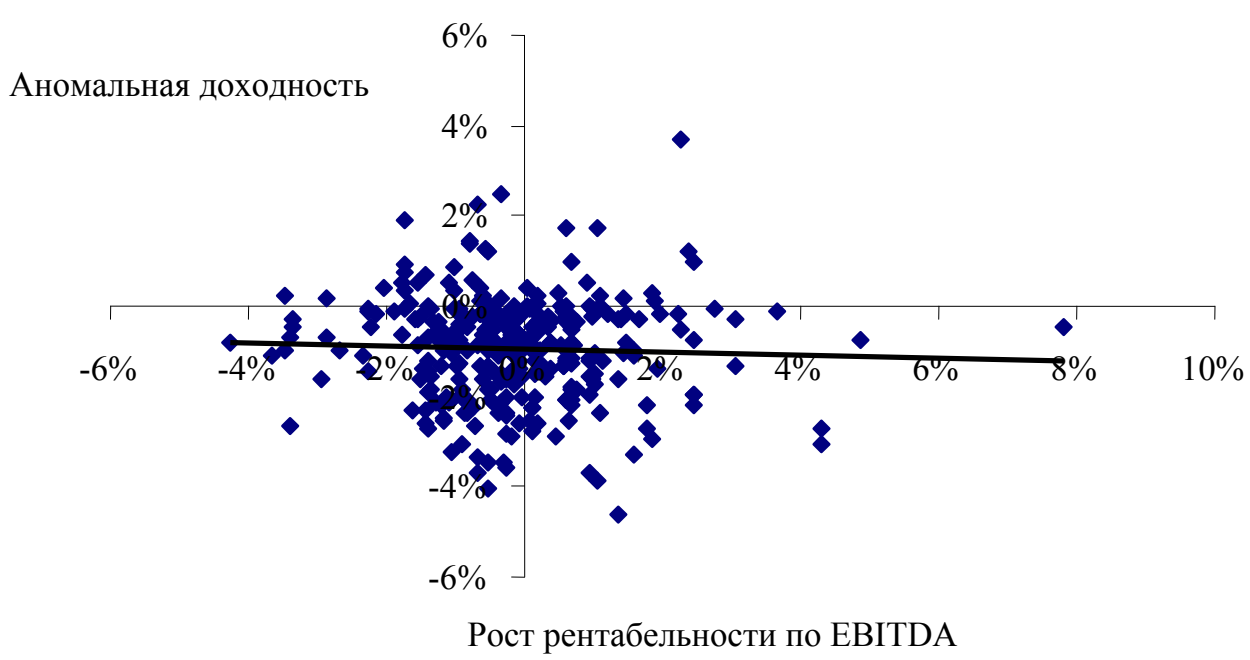

Рисунок 4. Регрессия между аномальной доходностью в однодневном временном окне и изменением маржи компании (полная выборка)

В случае изменения временного окна вновь проверяется аналогичная гипотеза.

Гипотеза Н0: существует значимая зависимость между аномальной доходностью и изменением рентабельности по EBITDA.

Полученные результаты проверки гипотезы при изменившемся временном окне приведены в таблице 9.

Таблица 9.

Краткая характеристика регрессии между аномальной доходностью в однодневном временном окне и изменением маржи компании

\begin{tabular}{|l|l|}
\hline $\mathrm{R}^{\wedge} 2$ & 0,0003 \\
\hline Скорректированный $\mathrm{R}^{\wedge} 2$ & $-0,003$ \\
\hline Стандартная ошибка & 0,014 \\
\hline F-статистика & $0,78>0$ \\
\hline Формула & $\mathrm{y}=-0,03 \mathrm{x}-1.5 \%$ \\
\hline
\end{tabular}

Тесты на значимость (F-тест) и устойчивость показывают незначимость и неустойчивость полученной регрессии. Гипотеза Н0 не подтверждается.

Регрессионный анализ показывает значительно меньшую зависимость между моментальной реакций рынка и последующими результатами сделки.

Мы считаем, что данный факт связан с тем, что, во-первых, не вся информация успевает дойти до всех участников рынка. Во-вторых, в столь короткий промежуток времени велика степень эмоциональной реакции мелких спекулятивных трейдеров. Для взвешенной реакции институциональных инвесторов требуется значительно большее время.

\section{V. Тестирование модели на подвыборках}

\section{1. Подвыборка по типам рынков капитала}

Разделение выборки на сделки на развитых и растущих рынках капитала дает возможность оценить степень оценки рынком эффекта от сделки. Ввиду того что доля публичных компаний на растущих рынках значительно меньше, нежели на развитых, выборки имеют разную мощность. 
Гипотеза Н0: существует значимая зависимость между аномальной доходностью и изменением рентабельности по EBITDA.

Результаты тестирования модели на этих подвыборках приведены в таблице 10.

Таблица 10.

Краткая характеристика выборки данных, тыс. долл.

\begin{tabular}{|l|l|l|}
\hline & \multicolumn{1}{|c|}{ Развитые рынки } & \multicolumn{1}{|c|}{$\begin{array}{c}\text { Растущие } \\
\text { рынки }\end{array}$} \\
\hline Количество наблюдений & 277 & 40 \\
\hline $\begin{array}{l}\text { Средняя выручка компании- } \\
\text { цели }\end{array}$ & 30109 & 114720 \\
\hline ЕВITDA компании-цели & 5822 & 22198 \\
\hline $\begin{array}{l}\text { Средняя рентабельность по } \\
\text { ЕВITDA }\end{array}$ & $24 \%$ & $24 \%$ \\
\hline Средняя аномальная доходность & $-1 \%$ & $-1 \%$ \\
\hline Регрессия & $\mathrm{y}=0,6 \mathrm{x}-1.5 \%$ & $\mathrm{y}=0,4 \mathrm{x}-1.5 \%$ \\
\hline $\mathrm{R}^{\wedge}$ & 0,4 & 0,3 \\
\hline
\end{tabular}

Тесты на значимость (F-тест) и устойчивость показывают, что результаты статистически значимы и устойчивы. Гипотеза Н0 подтверждается. Коэффициент детерминации показывает, что предсказательная точность рынка развитых стран $(0,4)$ выше, чем развивающихся $(0,3)$.

Регрессионный анализа на двух выборках, взятых раздельно, показал следующие результаты. Для развитых рынков они отражены на рисунке 5, а для растущих рынков капитала - на рисунке 6.

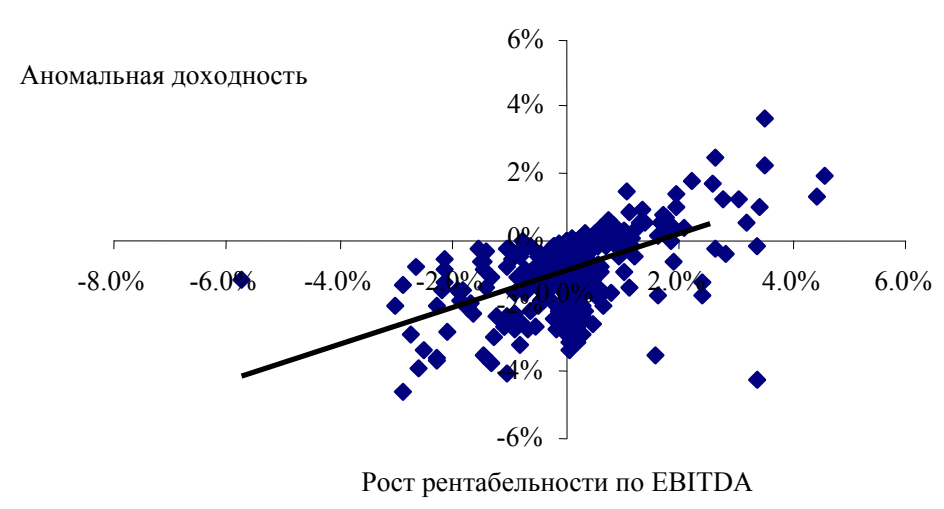

Рисунок 5. Регрессия между аномальной доходностью в однодневном временном окне и изменением рентабельности компании (развитые рынки) 


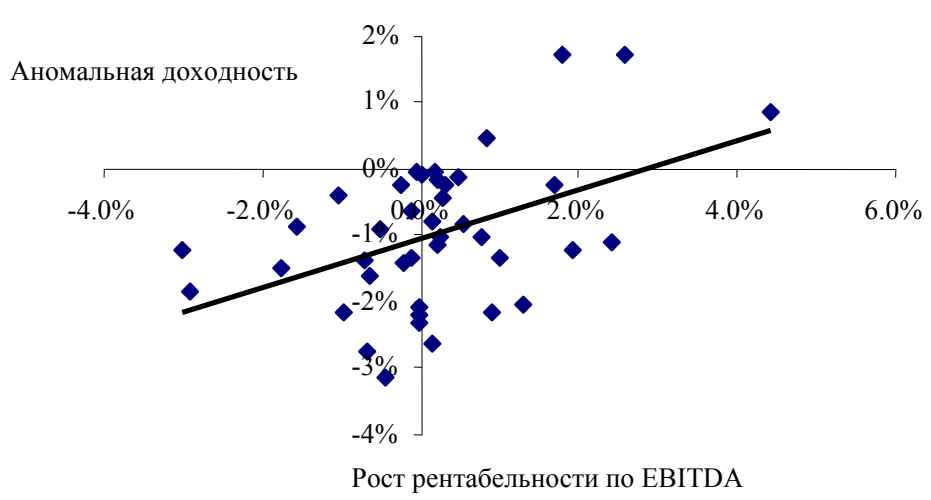

Рисунок 6. Регрессия между аномальной доходностью в однодневном временном окне и изменением рентабельности компании на растущих рынках

Мы считаем, что это объясняется более высокой эффективностью рынка развитых стран в целом, вызванное большим количеством игроков, большей прозрачностью и аналитическим покрытием.

Проведение стандартных тестов на однородность показывает, что, несмотря на меньшую точность, выборки являются однородными. Это подтверждает гипотезу, что степень эффективности рынков развивающихся стран близится к развитым.

\section{2. Подвыборка по размерам компаний}

Разделение выборки на крупнейшие и остальные компании позволяет косвенно оценить степень эффективности рынка. При этом к крупнейшим отнесены те поглощающие компании, выручка которых до сделки превышает \$10 млрд.

Гипотеза Н0: существует значимая зависимость между аномальной доходностью и изменением рентабельности по EBITDA.

Результаты тестирования для данных подвыборок приведены в таблице 11.

Таблица 11.

Краткая характеристика выборки данных, тыс. долл.

\begin{tabular}{|l|l|l|}
\hline & Крупнейшие & Остальные \\
\hline Количество наблюдений & 168 & 149 \\
\hline Средняя выручка компании-цели & 73338 & 4082 \\
\hline ЕВITDА компании-цели & 13992 & 1006 \\
\hline Средняя рентабельность по ЕВITDA & $21 \%$ & $28 \%$ \\
\hline Средняя аномальная доходность & $-1 \%$ & $-1 \%$ \\
\hline Регрессия & $\mathrm{y}=0,6 \mathrm{x}-1.5 \%$ & $\mathrm{y}=0,5 \mathrm{x}-1.5 \%$ \\
\hline $\mathrm{R}^{\wedge} 2$ & 0,51 & 0,22 \\
\hline
\end{tabular}

Тесты на значимость (F) и устойчивость показывают значимость и устойчивость полученной регрессии. Гипотеза Н0 подтверждается.

Графическое представление на рисунках 7 и 8 наглядно иллюстрирует связь результатов, измеренных бухгалтерскими и рыночными показателями, в случае анализа крупных поглощающих компаний относительно всех остальных из выборки. 


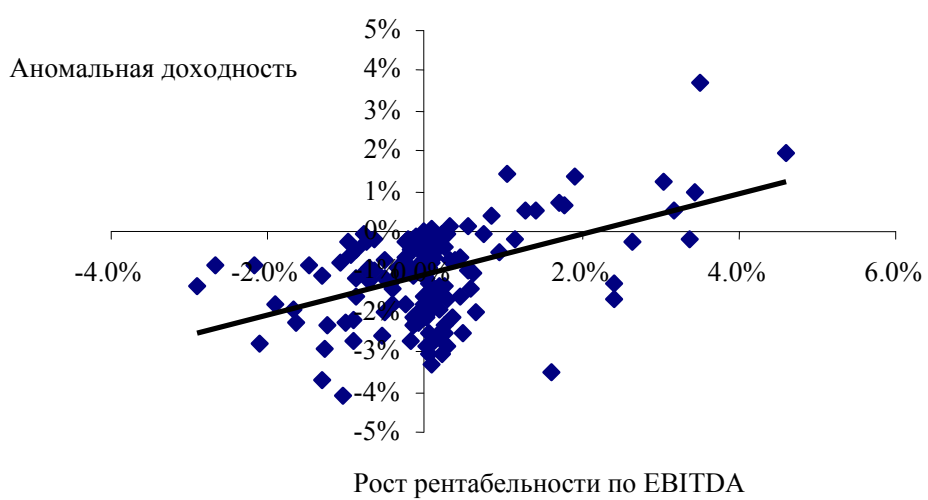

Рисунок 7. Регрессия между аномальной доходностью в однодневном временном окне и изменением рентабельности компании (маленькие компании)

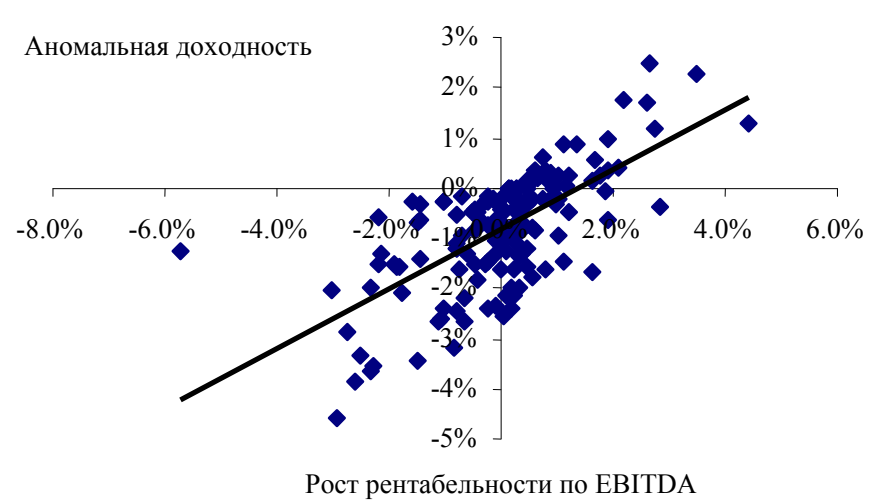

Рисунок 8. Регрессия между аномальной доходностью в однодневном временном окне и изменением рентабельности компании (крупнейшие компании)

Регрессионный анализ показывает, что коэффициент детерминации $(0,5)$ для крупнейших компаний выше, чем для остальных компаний $(0,2)$. Мы считаем, что это напрямую связано с гипотезой эффективного рынка. Поскольку акции крупнейших компаний, как правило, полнее и тщательнее отслеживаются аналитиками, то информация эффективней находит свое отражение в котировках

\section{Выводы}

Применение интегрированной методики, соединяющей бухгалтерские и рыночные показатели для измерения эффектов от сделок, на выборке сделок с 2001-го по 2004 год позволило выявить четкую взаимосвязь между краткосрочной реакцией рынка на новость о слиянии и реальной последующей эффективностью сделки. Применение различных статистических тестов подтверждает устойчивость полученных выводов.

Выводы не меняются при разделении выборки на крупнейшие и остальные компании, компании с развитых и растущих рынков капитала. Изолированные выборки имеют лишь различный уровень точности регрессии, что косвенным образом отражает различие в степени эффективности рынка. Тест на однородность показывает идентичность полученных регрессий для различных выборок данных.

Данные выводы не сохраняются при значительном уменьшении анализируемого временного окна относительно появления информации о сделке, а также изменения базы для измерения аномальной доходности. 
Данные результаты подтверждают существование определенного уровня эффективности рынка и способность рынка быстро и независимо предсказать эффективность сделки и ее будущее влияние на благосостояние акционеров.

Бесспорно, полученные в данной работе выводы в определенной степени обобщают выводы, полученные предыдущими исследователями, и дают интересный инструмент для практического анализа сделок. Высокая точность реакции рынка на эффективность сделки позволяет акционерам компаний контролировать целесообразность интеграционного процесса.

\section{Литература}

1. Beitel P., Schiereck D., and Wahrenburg M. Explaining the M\&A-success in European Bank Mergers and Acquisitions // Working Paper, University of Witten/Herdecke, Germany, January 2002.

2. Bruner R. An Analysis of Value Destruction and Recovery in the Alliance and Proposed Merger of Volvo and Renault // Journal of Financial Economics, № 1, January, 1999, 51:125-166.

3. Bruner R., Eades K.M. The Crash of the Revco LBO: The Hypothesis of Inadequate Capital // Financial Management, 21, № 1, Spring, 1992, pp: 35-49.

4. Dickerson A., Gibson H., Tsakalotos E. The Impact of Acquisitions on Company Performance: Evidence From a Large Panel of U.K. Firms //Oxford Economic Papers, 49, № 3, July, 1997. pp: $344-361$.

5. Eckbo E., Thorburn K.. Gains to Bidder Firms Revisited: Domestic and Foreign Acquisitions in Canada // Journal of Financial and Quantitative Analysis, 35, № 1, March 2000. pp: $1-25$.

6. Goergen M., Renneboog L.. Shareholder Wealth Effects of European domestic and CrossBorder Takeover Bids // European Corporate Governance Institute, Finance working paper, № 8, 2003.

7. Healy P., Palepu K., Ruback R. Does Corporate Performance Improve After Mergers? // Journal of Financial Economics, 31, № 2, April 1992. pp: 135-175.

8. Healy P., Palepu K., Ruback R. Which Takeovers are Profitable: Strategic of Financial? // Sloan Management Review, 38, № 4, Summer 1997. pp: 45-57.

9. Herman E., Lowenstein L. The Efficiency Effect of Hostile Takeovers // Knights, Raiders, and Targets, New York, 1988. pp: 211-240.

10. Hietala P., Kaplan S., Robinson D. What is the Price of Hubris? Using Takeover Battles to Infer Overpayments and Synergies // NBER Working Paper № W9264, October 2002.

11. Jarrell G., Poulsen A. The Returns to Acquiring Firms in Tender Offers: Evidence from Three Decades // Financial Management, 18, № 3, Autumn 1989. pp: 12-19.

12. Kaplan S., Weisbach M. The Success of Acquisitions: Evidence From Divestitures // Journal of Finance, 47, № 1, March, 1992. pp: 107-138.

13. Kuipers D., Miller D., Patel A. The Legal Environment and Corporate Valuation: Evidence from Cross- Border Mergers // Texas Tech University working paper, January 2003.

14. Loughran T., Vijh A. Do Long-Term Shareholders Benefit From Corporate Acquisitions? // Journal of Finance, 52, № 5, December 1997. pp: 1765-1790.

15. Lys, Thomas, Vincent L. An analysis of value destruction in AT\&T's acquisition of NCR // Journal of Financial Economics, 39, №2/3 October/November, 1995. pp: 353-378.

16. Maquieria C., Megginson W., Nail L. Wealth Creation versus wealth redistributions in pure stock-for-stock mergers // Journal of Financial Economics, 48, № 1, April 1998. pp: 3-33.

17. Meeks, G. Disappointing Marriage: A Study of the Gains From Merger // Cambridge University Press. Cambridge, 1977.

18. Mitchell M., Mulherin J.H., The Impact of Industry Shocks on Takeover and Restructuring Activity // Journal of Financial Economics, 41, 1996. pp: 193-229. 
19. Mueller D. Mergers and Market Share // Review of Economics and Statistics, 67, № 2, May 1985. pp: 259-267.

20. Mulherin J.H., Boone A.L. Comparing Acquisitions and Divestitures // Journal of Corporate Finance, 6, 2000. pp: 117-139.

21. Parrino J.D., Harris R.S. Business Linkages and Post-merger Operating Performance // Working Paper, Charlottesville VA: Darden Graduate School of Business, University of Virginia, 2001.

22. Ravenscraft D., Scherer F.M. Mergers, Sell-Offs, \& Economic Efficiency, The Brookings Institute, 1987, Washington D.C.

23. Richard A. Brealy, Stewart C. Myers Principles of corporate finance, 1991. p. 918

24. Ruback R. The Conoco takeover and stockholder returns // Sloan Management Review № 23, 1982. pp:13-33.

25. Servaes H. Tobin's Q and the Gains from Takeovers // Journal of Finance, 46, №1, March 1991. pp: 409-419.

26. Seth A. Sources of Value Creation in Acquisitions: An Empirical Investigation // Strategic Management Journal, 11, №6, 1990. pp: 431—446.

27. Sharma D., Ho J. The Impact of Acquisitions on Operating Performance: Some Australian Evidence // Journal of Business Finance and Accounting, № 29(1) January, March 2002. pp: $155-200$. 